\title{
A reference-dependent user equilibrium model for activity-travel scheduling
}

\author{
Qing $\mathrm{Li}^{1,2} \cdot$ Feixiong Liao ${ }^{2}$ - Harry J. P. Timmermans ${ }^{2}$ • \\ Jing Zhou ${ }^{1}$
}

Published online: 12 July 2016

(C) The Author(s) 2016. This article is published with open access at Springerlink.com

\begin{abstract}
Existing user equilibrium models of activity-travel scheduling generally fall short in representing travelers' decision-making processes. The majority have either implicitly or explicitly assumed that travelers follow the principle of utility maximization. This assumption ignores the fact that individuals may be loss-averse when making activity-travel decisions. Allowing for the situation that travelers possess accurate information of the urban-transportation system due to modern technologies, studies on reference-dependent decision-making under near-perfect information are receiving increasing attention. In view of traveler heterogeneity, individuals can be divided into multiple classes according to their reference points. In this paper, we propose a reference-dependent multiclass user equilibrium model for activity-travel scheduling, which can be reformulated as a variational inequality problem. Moreover, comparative analyses are conducted on the equilibrium states between utility-maximization (no reference) and reference-dependency of exogenous and endogenous references. A numerical example regarding combined departure-time and mode choice for commuting is conducted to illustrate the proposed model. The simulated results indicate that reference points and loss aversion attitudes have significant effects on the choice of departure time and mode.
\end{abstract}

Feixiong Liao

f.liao@tue.nl

Qing Li

liqing_321@hotmail.com

Harry J. P. Timmermans

h.j.p.timmermans@tue.nl

Jing Zhou

jzhou@nju.edu.cn

1 School of Management and Engineering, Nanjing University, Nanjing 210093, People's Republic of China

2 Urban Planning Group, Eindhoven University of Technology, PO Box 513, 5600 MB Eindhoven, The Netherlands 
Keywords Reference-dependency · Activity-travel scheduling · Heterogeneity · Loss aversion $\cdot$ Multi-state supernetwork

\section{Introduction}

Over the past decades, increasing attention has been paid to the activity-based modeling paradigm in the study of activity-travel behavior. Studies pertain to route choice, mode choice, departure time choice, activity and parking location choice, and the combination of two or more of these decisions. Recent reviews of activity-based approaches can be found in Pinjari and Bhat (2011) and Rasouli and Timmermans (2014). Along this line of research, network equilibrium models, which mostly adhere to Wardrop's first equilibrium principle, have been proposed for modeling the activity-travel scheduling problem at the aggregate level. Models of this kind are generally useful for long-term transport planning. Due to the scalability of the network representation, network equilibrium models offer advantages in studying the effects of policy interventions and other external factors on activity-travel decisions.

From an activity-based perspective, the so-called multi-state supernetwork representation, suggested in Arentze and Timmermans (2004) offers the most powerful, comprehensive representation as it allows the study of the simultaneous choice of all relevant facets underlying activity scheduling processes. Their multi-state supernetwork models can represent the full choice options and capture the various interdependences in activity-travel trip chaining. Recently, the original formalism has been improved and elaborated (Liao et al. 2010, 2011). Using extensions of the original model, time-dependency (Liao et al. 2013a), joint travel (Liao et al. 2013b), and activity-travel assignment (Liu et al. 2015) have been studied. Other similar representations have been suggested, but they do not offer the full representation of activity-travel patterns. Ramadurai and Ukkusuri (2010) studied the activity-travel scheduling problem based on a cell-based transmission model, in which the travel and activity links were represented as cells. Using a time-expanded multi-modal supernetwork, Li et al. (2010) investigated the timetabling problem of transit services. Ouyang et al. (2011) formulated an activity-based user equilibrium model with queued links in congested networks. Fu and Lam (2014) proposed an activity-based network equilibrium model considering the stochasticity of activity utilities.

As far as the choice mechanisms are concerned, most equilibrium models for activitytravel scheduling assume that travelers follow the principle of utility-maximization. However, there is a large body of field and experimental evidence which suggests that decision-making processes involve reference-dependent choice mechanisms, in which individuals identify gains and losses relative to reference points. Reference-dependent theory under risk or uncertainty originated from well-known (cumulative) prospect theory proposed by Kahneman and Tversky (1979), in which only one attribute of alternatives was considered. Later, they (Tversky and Kahneman 1991) extended the decision-making problem to the multi-attribute case under certainty. With the ever-increasing power of modern technologies, travelers have more accurate and relatively perfect information about the urban systems. Thus, the activity-travel scheduling problem can be conceptualized as a reference-dependent choice-making process under certainty. A few studies have incorporated reference-dependency into static traffic assignment problems. De Borger and Fosgerau (2008) have conducted experimental validations of reference-dependent model of route choice. Delle Site and Filippi (2011) and Delle Site et al. (2013) applied stochastic 
user equilibrium models with exogenous and endogenous reference points. However, there is a gap in the formulation of reference-dependent activity-based dynamic traffic assignment problems.

Therefore, this study proposes a reference-dependent user equilibrium model under certainty (no risk) for activity-travel scheduling. It enriches a growing body of literature of integrating activity-travel scheduling and dynamic traffic assignment by incorporating a more behaviorally sound choice-making mechanism. The remainder of the paper is organized as follows. "Reference-dependent theory and equilibrium analysis" section introduces the reference-dependent theory and the process of the activity-based user equilibrium problem. "Model assumptions and definitions" section discusses the model assumptions and the definitions of link utilities. "Reference-dependent user equilibrium model" section presents the proposed reference-dependent model with heterogeneous travelers of different references. The solution algorithm for the proposed model is discussed in "Solution algorithm" section. A numerical example with simulated scenarios is provided in "Numerical example" section to illustrate the proposed model. Finally, conclusions and a plan of future research are drawn in the last section.

\section{Reference-dependent theory and equilibrium analysis}

\section{Reference-dependent theory}

The reference-dependent theory under no risk proposed by Tversky and Kahneman (1991) was an extension in the consideration of multi-attributes of prospect theory under uncertainty studied in Kahneman and Tversky (1979). The trials conducted in their work were used to examine some anomalous phenomena, such as endowment effects, status quo bias and the gap between willingness to pay and willingness to accept. Traditional expected utility theory cannot provide a proper interpretation for those phenomena. Referencedependent theory incorporates the following features:

(1) Reference dependence people distinguish between gains from losses before making decisions; the outcomes are framed as gains or losses compared to some reference points;

(2) Loss aversion losses (outcomes below the reference point) loom larger than the corresponding gains (outcomes above the reference point);

(3) Constant loss aversion a certain loss on one dimension needs corresponding multifold gains to compensate in the same dimension.

Assuming the loss aversion is constant, the utility function for an alternative of two attributes with $r$ as the reference point satisfying the above features can be represented as follows:

$$
\begin{gathered}
U_{r}\left(x_{1}, x_{2}\right)=U\left(R_{1}\left(x_{1}\right), R_{2}\left(x_{2}\right)\right) \\
R_{i}\left(x_{i}\right)=\left\{\begin{array}{ll}
u_{i}\left(x_{i}\right)-u_{i}\left(r_{i}\right), & x_{i} \geq r_{i} \\
\lambda_{i}\left(u_{i}\left(r_{i}\right)-u_{i}\left(x_{i}\right)\right), & x_{i}<r_{i}
\end{array}, \quad i=1,2\right.
\end{gathered}
$$

The functions $R_{i}($.$) are the value functions associated with the reference point. x_{i}$ and $r_{i}$ are the outcome and reference value of an alternative of the $i$-th attribute respectively. $\lambda_{i}$ is interpreted as a coefficient of loss aversion for the $i$-th attribute. If, for example, 
$\lambda_{1}>\lambda_{2}>1$, it means that individuals are more sensitive to the losses in attribute 1 than in attribute 2 .

\section{User equilibrium of reference-dependent activity-travel patterns}

Activity-travel scheduling involves activity-travel choices in the travel demand domain related to transport network service provisions within the supply domain. On the travel demand side, endeavors from the travel behavior research community have been aimed at modeling activity-travel decisions. The primary outputs are time-dependent activity-travel patterns at the disaggregate level. Individuals' decision-making behavior determines the distribution of travel demand and thus traffic flows in transport networks. Based on the possible reference points, individuals experience the results of their decisions on the transport network, and these travel experiences may in turn influence their reference points. This can lead to subsequent rescheduling of their travel patterns. The traffic flows on the network can be seen as the result of an activity-travel scheduling process, which is a temporal and spatial representation of activity-travel engagement decisions. The reference points evolve with the traffic flows in a "day-to-day" dynamic process. The long-term equilibrium is achieved when no single traveler can increase his/her path utilities by unilaterally changing his/her travel patterns. Hence, the final state is consistent with the idea of the first Wardropian principle. The process is depicted in Fig. 1.

\section{Model assumptions and definitions}

The user equilibrium model in this study is examined in a simplified multi-state supernetwork through which any path corresponds to an activity-travel pattern, consisting of time-dependent activity and travel links (Liao et al. 2013a). A simple example of a multistate supernetwork is shown in Fig. 2, which integrates an individual's activity program (only one activity is shown for simplicity), a multi-modal transport network, and the

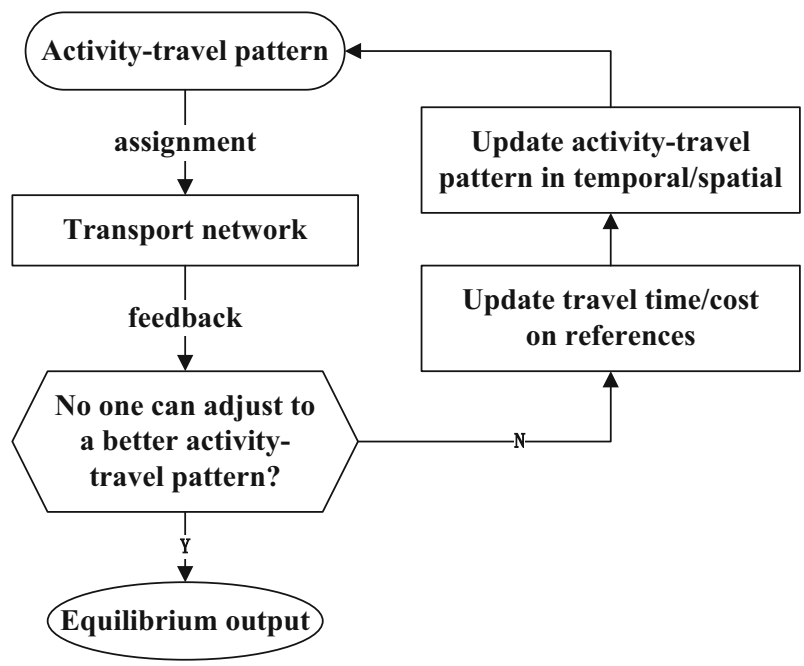

Fig. 1 Equilibrium process of reference-dependent activity-travel scheduling 


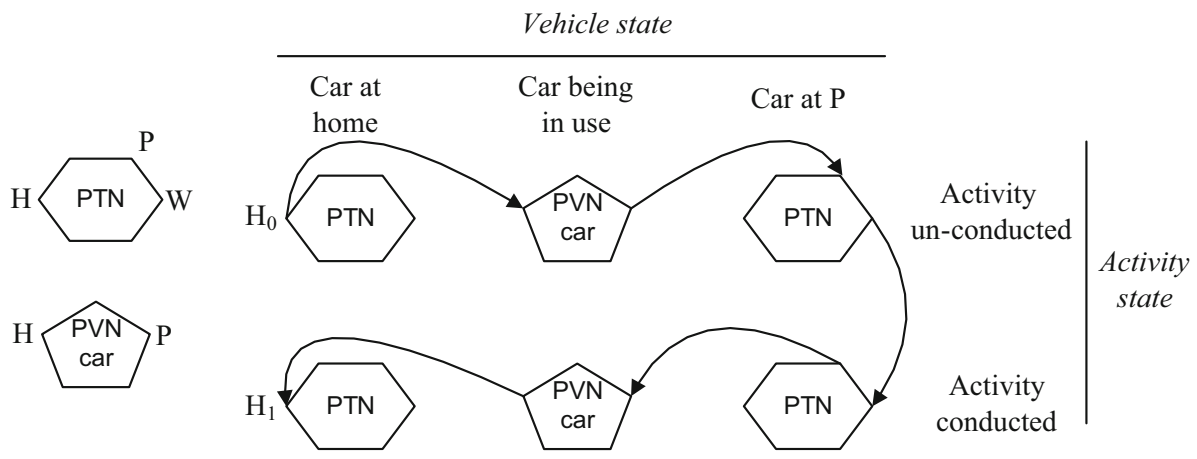

Fig. 2 A small example of multi-state supernetwork

locations of facilities. The pentagon denotes a private vehicle network (PVN), which can only be accessed by one type of private vehicle; the hexagon denotes a public transport network (PTN), in which the individual can walk and take public transport. To capture the choice space of conducting the activity, these networks are assigned to and connected by different states of the implementation of the activity program. A feature is that any path through this multi-state supernetwork expresses a particular way of conducting the activity. As an example, the route from $\mathrm{H}_{0}$ to $\mathrm{H}_{1}$ represents the tour characterized by the individual leaving home by car, parking the car at $\mathrm{P}$, taking public transport connection to conduct activity at $\mathrm{W}$, and then going back to $\mathrm{P}$ and returning home by car.

In order to facilitate the essential ideas without loss of generality, the assumptions adopted for the proposed model are discussed below.

\section{Model assumptions}

A1 The activity-travel scheduling problem is studied in a fixed time horizon $[0, T]$, divided into a finite set of time intervals, $\{t: t=1,2, \ldots, \bar{T}\}$. Let $\Delta$ be the length of the interval so that $\Delta \cdot \bar{T}=T$.

A2 Ageneralized reference-dependent utility is used to represent the amount of utility derived from travel and activity participation. The activity-travel path utility is the sum of utilities of all the connected links. The travelers' scheduling problem is to choose a feasible path consisting of travel and activity links based on the path utility.

A3 Due to modern technologies, the attributes of travel alternatives are known in real time. Travelers have perfect information of the transport network. They maximize their reference-dependent utility.

A4 Travelers are loss-averse regarding travel choices with multiple attributes. Deterministic travel time and monetary costs are the two main factors underlying travel utility (the generalization to a richer set of factors is straightforward). Due to the complexity of context-dependency for activity participation, we do not consider the reference-dependency for activity utility. 


\section{Link utilities}

Let $G(N, A)$ be the multi-state supernetwork constructed for conducting a certain daily activity program, where activity-travel patterns always start and end at home. $N$ denotes the location set in space, and $A$ is the link set $\left(A=A_{t} \cup A_{a}\right)$, including travel links $\left(A_{t}\right)$ and activity links $\left(A_{a}\right)$. An activity link means conducting an activity. Travel links $\left(A_{t}=A_{t}^{b} \cup A_{t}^{c} \cup A_{t}^{s}\right)$ represent the travel episodes between two successive activity locations involving three different modes, namely, taking bus $\left(A_{t}^{b}\right)$, driving by car $\left(A_{t}^{c}\right)$, and taking subway $\left(A_{t}^{s}\right)$. For each travel link, each class of travelers has a reference point. Suppose travelers are divided into $|M|$ classes externally according to distinct references, where $M$ is the set of traveler classes and $m$ is a member of $M$.

\section{Reference-dependent utility of travel links}

Given assumption A4, we can specify the reference-dependent model. Suppose the utility of travel links depends on two attributes: travel time expenditure and monetary cost. Gains and losses in the two attributes are defined relative to a reference point; the utility increases with gains and decreases with losses, and the change is steeper for losses than for gains. The reference-dependent utility for each travel link during time period $t$ is defined as (Delle Site and Filippi 2011; Delle Site et al. 2013), $a \in A_{t}, m \in M$.

$$
\begin{gathered}
T U_{a}^{m}(t)=\beta_{G T} G T_{a}^{m}(t)+\beta_{G M} G M_{a}^{m}(t)+\beta_{L T} L T_{a}^{m}(t)+\beta_{L M} L M_{a}^{m}(t) \\
G T_{a}^{m}(t)=\max \left(T_{s e}^{m}(t)-T_{a}^{m}(t), 0\right) \\
L T_{a}^{m}(t)=\max \left(T_{a}^{m}(t)-T_{s e}^{m}(t), 0\right) \\
G M_{a}^{m}(t)=\max \left(M_{s e}^{m}(t)-M_{a}^{m}(t), 0\right) \\
L M_{a}^{m}(t)=\max \left(M_{a}^{m}(t)-M_{s e}^{m}(t), 0\right)
\end{gathered}
$$

where $T U_{a}^{m}(t)$ is the travel utility of class $m$ arriving at link $a$ during $t, G T_{a}^{m}(t)$ and $G M_{a}^{m}(t)$ are the gains in travel time and money respectively, $L T_{a}^{m}(t), L M_{a}^{m}(t)$ are losses in time and money respectively. $\beta_{G T}$ and $\beta_{G M}$ are gain coefficients, while $\beta_{L T}$ and $\beta_{L M}$ are loss coefficients. Since the travel utility increases with the gains and decreases with losses, we have $\beta_{G T}, \beta_{G M}>0, \beta_{L T}, \beta_{L M}<0$. Moreover, as travelers are loss averse, they are more sensitive to a loss than a gain. Hence, the loss coefficients loom larger than the gain coefficients for each attribute, i.e. $\left|\beta_{G T}\right|<\left|\beta_{L T}\right|,\left|\beta_{G M}\right|<\left|\beta_{L M}\right|$. Although the travel utility is assumed linearly, nonlinear forms may also be used. $T_{s e}^{m}(t)$ and $M_{s e}^{m}(t)$ are the reference points of time and money respectively for class $m$ on link $a$, which is the connection from location $s$ to $e$ during time period $t . T_{a}^{m}(t)$ and $M_{a}^{m}(t)$ are the travel time and money spent on link $a$ of class $m$ arriving at link $a$ during $t$.

Corresponding to the above definitions, the time and money spent on each travel link are further specified as follows:

$$
\begin{gathered}
T_{a}^{m}(t)=t_{a}^{0}\left(1+\eta_{0}\left(\frac{F_{a} \cdot E_{a}+x_{a \in A_{t}^{c}}(t)}{C_{a}}\right)^{\eta_{1}}\right), \quad M_{a}^{m}(t)=\tau_{a}, \quad a \in A_{t}^{b} \\
T_{a}^{m}(t)=t_{a}^{0}\left(1+\eta_{0}\left(\frac{\left.F_{a} \cdot E_{a}\right|_{a \in A_{t}^{b}}+x_{a}(t)}{C_{a}}\right)^{\eta_{1}}\right), \quad M_{a}^{m}(t)=\theta_{a} \cdot T_{a}^{m}(t), \quad a \in A_{t}^{c}
\end{gathered}
$$




$$
T_{a}^{m}(t)=t_{a}^{0}, \quad M_{a}^{m}(t)=\tau_{a}, \quad a \in A_{t}^{s}
$$

Equations (8)-(10) are the travel time and money expenditure for bus, car and subway respectively, where $\eta_{0}$ and $\eta_{1}$ are parameters of the BPR (Bureau of Public Road) function which can be estimated from historical data on traffic flows and travel times of the links. $t_{a}^{0}$ is the free flow travel time on link $\alpha$, which varies with travel mode. Let $F_{\alpha}$ be the fixed frequency of the bus and $E_{a}$ the number of passenger cars equivalents for a bus vehicle (Lo et al. 2003) during each interval. Since buses usually follow a fixed schedule, they contribute a constant effect to the traffic volume in each period. $x_{a \in A_{t}^{c}}(t)$ is the traffic flow on travel link $a\left(a \in A_{t}^{c}\right)$ or the number of car travelers during time period $t$. Considering bus and car share the same roads with the same link capacity $C_{a}$, the congestion effect are the same on both link $a \in A_{t}^{b}$ and link $a \in A_{t}^{c}$. The travel time of subway is fixed. $\tau_{a}$ is the fare of taking bus or subway, which is constant for one trip. Travel monetary cost by car is simply assumed to be a linear function of travel time, and $\theta_{a}$ is the coefficient $\left(\theta_{a}>0\right)$.

\section{The utility of activity links}

Given activity time window constraints, the utility of conducting an activity consists of the utility derived from activity participation and the penalty from arriving early and late. In this paper, Ettema and Timmermans' (2003) bell-shaped marginal utility function is adopted to express the utility of activity participation. This choice is in line with several previous studies, including Li et al. (2010), Ouyang et al. (2011), and Fu and Lam (2014), etc. The utility, $A U_{a}^{m}(t)$, derived by a traveler who arrives at activity link $a$ at $t$ can be defined as

$$
\begin{gathered}
A U_{a}^{m}(t)=S D_{a}^{m}(t)+\int_{t}^{t+d_{a}^{m}(t)} M U_{a}(\omega) d \omega, a \in A_{a} \\
S D_{a}^{m}(t)=\beta_{a}^{L T, e} \max \left(t_{a}^{s *}-\Delta_{a}^{m}-t, 0\right)+\beta_{a}^{L T, l} \max \left(t-t_{a}^{s^{*}}-\Delta_{a}^{m^{\prime}}, 0\right), \quad a \in A_{a} \\
M U_{a}(\omega)=\frac{\gamma_{a} \beta_{a} u_{a}^{\max }}{\exp \left[\beta_{a}\left(\omega-\alpha_{a}\right)\right]\left\{1+\exp \left[-\beta_{a}\left(\omega-\alpha_{a}\right)\right]\right\}^{\gamma_{a}+1}}, \quad a \in A_{a}
\end{gathered}
$$

where $S D_{a}^{m}(t)$ is the disutility of schedule delay of class $m$ who arrive at link $a$ at $t ; \beta_{a}^{L T, e}$ and $\beta_{a}^{L T, l}$ are the value of one unit time loss of early arrival and late arrival on activity link a. $M U_{a}(\omega)$ is the marginal utility gained from a time unity of participation at time $\omega . \alpha_{a}, \beta_{a}$ and $\gamma_{a}$ are activity-specific parameters, which can be estimated from survey data. $d_{a}^{m}(t)$ is the activity duration. Let $\left[t_{a}^{s}, t_{a}^{e}\right]$ be the time window on activity link $a$, and $\left[t_{a}^{s *}-\Delta_{a}^{m}, t_{a}^{s^{*}}+\Delta_{a}^{m \prime}\right]\left(\Delta_{a}^{m} \geq 0, \Delta_{a}^{m^{\prime}} \geq 0\right)$ be the desired interval of arrival time on link $a$ of class $m$ (Lam et al. 2006). $\Delta_{a}^{m}$ and $\Delta_{a}^{m \prime}$ are the duration tolerance of arriving earlier and later than the ideal start time $t_{a}^{s *} . t<t_{a}^{s *}-\Delta_{a}^{m}$ means arriving too earlier, causing a loss of time; $t>t_{a}^{s *}+\Delta_{a}^{m \prime}$ means late arrival, which also causes a loss of time (Senbil and Kitamura 2004). However, the utility loss per unit time of late arrival is larger than that of early arrival, which means $\left|\beta_{a}^{L T, e}\right|<\left|\beta_{a}^{L T, l}\right|$. When the arrival time $t$ is inside the desired interval i.e., $t_{a}^{s *}-\Delta_{a}^{m} \leq t \leq t_{a}^{s *}+\Delta_{a}^{m \prime}$, there is no gain and no loss in time so that $S D_{a}^{m}(t)$ equals to 0 . 


\section{Reference-dependent user equilibrium model}

In this section, we present the reference-dependent user equilibrium model for activitytravel scheduling. The constraints for traffic flows of the network in our study are formulated as below. These constraints include flow conservation for links and nodes, flow propagation, definitional constraints, non-negativity conditions and boundary conditions. In addition, the model formulation and two other scenarios to study the effects of references are given.

\section{Network flow constraints}

Let $x_{a k}^{m p}(t), u_{a k}^{m p}(t)$ and $v_{a k}^{m p}(t)$ be the traffic flow, inflow and outflow of class $m$ on link $a$ during $t$ that depart from home and enter path $p \in P$ during $k$ respectively. $f_{p}^{m}(k)$ is the departure flow of $m$ from home by path $p$ during interval $k, k \in[0, T] . x_{a}(t), u_{a}(t)$ and $v_{a}(t)$ are the cumulative traffic flow, inflow and outflow on link $a$ during $t$. Following (Huang and Lam 2002; Liu et al. 2015), the constraint set for the reference-dependent user equilibrium model for multi-class activity-travel scheduling problem can be summarized as follows.

Flow conservation constraints:

$$
\begin{gathered}
\sum_{a \in E n(i)} \sum_{p} \sum_{k} u_{a k}^{m p}(t)=\sum_{a \in E x(i)} \sum_{p} \sum_{k} v_{a k}^{m p}(t), \quad \forall m, t \\
x_{a k}^{m p}(t+1)=x_{a k}^{m p}(t)+u_{a k}^{m p}(t)-v_{a k}^{m p}(t), \quad \forall a, k, m, p \\
u_{a k}^{m p}(t)=\delta_{a k}^{m p}(t) \cdot f_{p}^{m}(k), \quad \forall m, \quad p, k
\end{gathered}
$$

where $E n(i)$ and $E x(i)$ are the link sets whose entrance node and exit node are $i$ respectively. $\delta_{a k}^{m p}(t)$ is an indicator variable, if class $m$ enters path $p$ from home during $k$ and arrives at $a$ during $t$, the variable will be 1 ; otherwise, 0 .

Flow propagation constraints:

$$
v_{a k}^{m p}\left(t+\operatorname{INT}\left(\frac{T_{a}^{m}(t)}{\Delta}+0.5\right)\right)=u_{a k}^{m p}(t), \quad \forall m, p, a, k
$$

Equation (17) depicts that travelers departing from home during $k$ by $p$ and arrive at $a$ during $t$ will exit $a$ during $t+\operatorname{INT}\left(\frac{T_{a}^{m}(t)}{\Delta}+0.5\right)$. For $a \in A_{a}, T_{a}^{m}(t)$ is the activity duration of $m$ travelers who arrive at activity link $a$ during $t$.

Definitional constraints:

$$
\begin{aligned}
\sum_{m} \sum_{p} \sum_{k} u_{a k}^{m p}(t) & =u_{a}(t), \forall a \\
\sum_{m} \sum_{p} \sum_{k} v_{a k}^{m p}(t) & =v_{a}(t), \quad \forall a \\
\sum_{m} \sum_{p} \sum_{k} x_{a k}^{m p}(t) & =x_{a}(t), \quad \forall a
\end{aligned}
$$


Non-negativity conditions:

$$
x_{a k}^{m p}(t) \geq 0, \quad u_{a k}^{m p}(t) \geq 0, \quad v_{a k}^{m p}(t) \geq 0, \quad \forall m, p, a, k
$$

Boundary conditions:

$$
\begin{gathered}
\sum_{m} \sum_{p} \sum_{k} f_{p}^{m}(k)=q \\
\sum_{p} \sum_{k} f_{p}^{m}(k)=q^{m}, \quad \forall m
\end{gathered}
$$

where the total demand for activity-travel is $q$, and $q^{m}$ is for class $m$. The utility gain from the daily activity-travel pattern $p$ can be obtained by summing the link utilities.

$$
U_{p}^{m}(k)=\sum_{a \in A_{t}} \sum_{t} \delta_{a k}^{m p}(t) \cdot T U_{a}^{m}(t)+\sum_{a \in A_{a}} \sum_{t} \delta_{a k}^{m p}(t) \cdot A U_{a}^{m}(t), \quad \forall m, p
$$

\section{Model formulation}

According to assumption A3, individuals from each class will schedule their activity-travel patterns based on the reference-dependent utilities derived from a feasible path through the network. For each class, the utilities of all used paths are the largest and equal, and those of unused paths are smaller as discussed in "Reference-dependent theory and equilibrium analysis" section. Therefore, the scheduling problem is equivalent to finding the optimal path flow vector $f^{*}$ for each class satisfying constraints (14)-(23) such that the following conditions hold:

$$
\left\{\begin{array}{ll}
U_{p}^{m}(k)=U_{\text {max }}^{m}(k), & \text { iff } f_{p}^{m}(k)>0 \\
U_{p}^{m}(k) \leq U_{\text {max }}^{m}(k), & \text { iff } f_{p}^{m}(k)=0
\end{array}, \quad \forall m, p\right.
$$

where $U_{\text {max }}^{m}(k)=\max \left\{U_{p}^{m}(k)\right\}$.

The conditions can be formulated as an equivalent variational inequality (VI) problem as follows.

$$
\sum_{m} \sum_{p} \sum_{k} U_{p}^{m *}(k)\left(f_{p}^{m *}(k)-f_{p}^{m}(k)\right) \geq 0
$$

for all $f_{p}^{m}(k) \in\left\{\mathbf{f}: \sum_{m} \sum_{p} \sum_{k} f_{p}^{m}(k)=q, \sum_{p} \sum_{k} f_{p}^{m}(k)=q^{m} ; f_{p}^{m}(k) \geq 0, \forall m, p, k\right\}$, which is a compact convex set. The path utility function of class $m$ is continuous with the traffic flow but non-linear and non-convex. Thus, it can be proven that there is at least one solution for the problem (Huang and Lam 2002), while the uniqueness of solutions remains to be further verified.

\section{Analysis of the reference points}

Travelers' trade-off behavior between travel time and monetary cost can be described in terms of the reference-dependent framework. Different reference points will lead to difference results. The reference points in the proposed equilibrium model can be timedependent and endogenous (Scenario 0), evolving with the dynamic traffic flow patterns. 
Given a time interval and a trip between two locations, each class of travelers has a specific reference transport mode, and its reference point is formed by the travel time and cost of that transport mode according to the realization of the traffic flows in the network.

Scenario $O$ Reference points are time-dependent and endogenous based on the actual experiences of the current iteration in the day-to-day dynamic process.

To facilitate the influence of the reference point on the scheduling process, two scenarios further come into perspective.

Scenario 1 If there is no reference-point, the model will reduce to the standard generalized cost model. The utility for travel links will be similar to the definition given in Liao et al. (2011). That means,

$$
\begin{gathered}
T U_{a}^{m}(t)=-\beta_{t} T_{a}^{m}(t)-\beta_{m} M_{a}^{m}(t) \\
T_{s e}^{m}(t)=M_{s e}^{m}(t)=0
\end{gathered}
$$

where the coefficients $\beta_{t}>0$ and $\beta_{m}>0$ represent the weights of the time and money components by different modes, respectively. Correspondingly, the penalty of arriving too early can be set as $\beta_{a}^{L T, e}=\beta_{a}^{e}<0$ and $\beta_{a}^{L T, l}=\beta_{a}^{l}<0$.

As discussed in Delle et al. (2013), the reference point can also be treated as exogenous. It is exogenous when it is assumed to be independent of the current travel conditions over the network, such as the free-flow travel time and monetary cost on the shortest route. It assumes that travelers have the same references on each attribute between the same OD pair all the time.

Scenario 2 If the reference points are fixed all the time, the model $\left(a \in A_{t}, m \in M\right)$ will reduce to:

$$
\begin{gathered}
T U_{a}^{m}(t)=\beta_{G T} G T_{a}^{m}(t)+\beta_{G M} G M_{a}^{m}(t)+\beta_{L T} L T_{a}^{m}(t)+\beta_{L M} L M_{a}^{m}(t) \\
G T_{a}^{m}(t)=\max \left(T_{s e}^{m}-T_{a}^{m}(t), 0\right) \\
L T_{a}^{m}(t)=\max \left(T_{a}^{m}(t)-T_{s e}^{m}, 0\right) \\
G M_{a}^{m}(t)=\max \left(M_{s e}^{m}-M_{a}^{m}(t), 0\right) \\
L M_{a}^{m}(t)=\max \left(M_{a}^{m}(t)-M_{s e}^{m}, 0\right)
\end{gathered}
$$

where the reference points in travel time and monetary cost are static and constant for all travel links.

\section{Solution algorithm}

Since the time period is subdivided into $T / \Delta$ time intervals, the VI problem is a discrete time VI problem. We adopt the route-swapping method to solve our model (Nagurney and Zhang, 1997), which is widely used for path-based user equilibrium models (Huang and Lam 2002; Szeto and Lo 2006; Ramadurai and Ukkusuri 2010; Liu et al. 2015). After initializing the path flow and parameters of the utility functions, the activity-travel pattern utility is computed, based on which the traffic flows are updated until the left side of Eq. (26) meets a predefined stopping criterion. Given the property of forced convergence 
to a stopping criterion, we claim the achieved equilibrium state is simulated. The details are described as follows.

Step 0 Initialization.

Load the network, and set the parameters of the BPR functions and activity utility function. Initialize the link characteristics, reference points and travel demand of each class. Find an initial feasible solution $\left\{f_{p}^{m 0}(k)\right\}$. The iteration counter $l$ is set as 0 ;

Step 1 Relaxation

Find a new solution of the reference-dependent activity-travel scheduling model.

Step 1.1 Calculation Calculate the travel time and monetary cost for each link based on the current traffic flow; and then get both reference-dependent travel utilities and activity utilities using Eqs. (3)-(13) and the path utility by Eq. (24);

Step 1.2 Update the current traffic flow Compute

$$
f_{p}^{m}(k)_{l+1}= \begin{cases}\max \left(0, f_{p}^{m}(k)_{l}-\rho_{l} \cdot f_{p}^{m}(k)_{l} \cdot\left(U_{\max }^{m}(k)_{l}-U_{p}^{m}(k)_{l}\right),\right. & \text { if } p, k \notin \Omega_{l}^{m} \\ f_{p}^{m}(k)_{l}+\sum_{p} \sum_{k} \frac{f_{p}^{m}(k)_{l}-f_{p}^{m}(k)_{l+1}}{\left|\Omega_{l}^{m}\right|}, & \text { if } p, k \in \Omega_{l}^{m}\end{cases}
$$

where $\Omega_{l}^{m}=\left\{U_{\text {max }}^{m}(k)_{l}=U_{p}^{m}(k)_{l}, \forall p, k\right\}, \rho_{l}=\rho / \operatorname{INT}\left(\frac{l+1}{\mu}+0.5\right)(\rho$ and $\mu$ are predefined parameters).

Step 2 Convergence check for iterations. If

$$
\frac{\sum_{m} \sum_{p} \sum_{k} f_{p}^{m}(k)_{l} \cdot\left(U_{\text {max }}^{m}(k)_{l}-U_{p}^{m}(k)_{l}\right)}{\sum_{m} \sum_{p} \sum_{k} f_{p}^{m}(k)_{l} \cdot U_{m a x}^{m}(k)_{l}} \leq \varepsilon
$$

then stop, where $\varepsilon$ is the convergence tolerance. The current traffic flow satisfies the conditions of simulated user equilibrium; otherwise, set $l=l+1$, and go to Step 1 .

\section{Numerical example}

In this section, a numerical example is presented to illustrate the proposed model and solution algorithm. Specifically, we examine how travelers' loss aversion attitude and reference point affect their travel choices.

\section{Basic setting}

For illustration purpose, the network scale of the example is set small, and thus the activitytravel paths are enumerated. To apply the model in large-scale networks, representative and heavily reduced activity-travel paths for conducting an activity program must be generated. This can be done in multiple ways: (1) pre-selection of locations for activities and parking (e.g., Liao et al. 2011); (2) multi-modal trip choice generation (e.g., Bovy and Hoogendoorn-Lanser 2005); (3) applying lower but realistic time resolution (e.g., Liao 2016). Figure 3 shows a simple multi-modal network consists of two location zones (residential and workplace) and three transport mode alternatives, i.e., subway, bus and car. As shown, there are five activity-travel paths in total. Travel links by bus and car share the same physical road. The time window and the desired arrival time window of the work activity is set as [9:00 a.m., 17:00 p.m.] and [9:00 a.m., 9:00 a.m.] respectively. The study period is set from 06:00 a.m. to 20:00 p.m. and equally divided into 168 intervals (5 min per interval). The total number of potential travelers in the residential zone is given as 
Fig. 3 The example network

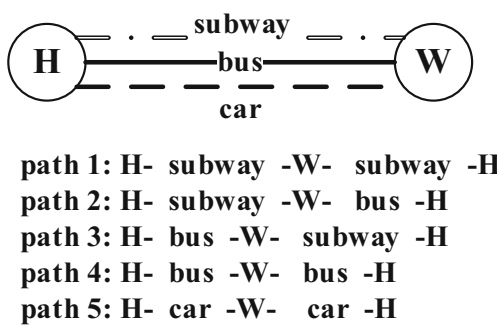

$q=3000$, which are divided into three classes based on distinct reference categories. Subway, bus and car are the reference mode for the first, second and third class of travelers respectively. $q^{m}$ and other parameters are set as follows: $q^{1}=q^{2}=q^{3}=1000, \beta_{G T}=$ $0.001, \beta_{L T}=-0.005, \beta_{G M}=0.001, \beta_{L M}=-0.005$ and $\beta_{a}^{L T, e}=-0.005, \beta_{a}^{L T, l}=-1.5$.

Table 1 shows the setting of gain and loss directions of choosing mode alternatives against the reference modes. The modes in first column denote the reference modes, each of which corresponds to a class of travelers. For the first class of travelers, choosing bus means a loss of time and gain in money, while choosing car means a gain in time and a loss of money. For the second class of travelers whose reference mode is bus, taking the subway or car both indicate a gain in time and loss of money, but the former is closer to the reference category. For the third class of travelers, taking the subway or bus both mean a loss of time and gain in money, and the two alternatives are close to each other. In accordance with Fig. 3, the reference paths of the three classes are path 1, path 4 and path 5 respectively.

Table 2 gives the link attributes of the links. For public transit, the reference-dependent utility experienced by travelers is not consistent with the actual travel time due to crowding discomfort. Crowding discomfort for subway and bus are modeled as follows (Lo et al. 2003).

$$
T T_{a}^{m}(t)=T_{a}^{m}(t)\left(1+0.1\left(\frac{x_{a k}^{m p}(t)}{C_{a}}\right)^{2}\right), \quad a \in A_{t}^{b} \cup A_{t}^{s}
$$

where $T T_{a}^{m}(t)$ is the modified in-vehicle travel time by bus and subway. The capacities are

Table 1 Alternative analysis for each class

Table 2 Travel link attributes (1 $\mathrm{RMB} \approx 0.152$ US dollar)

\begin{tabular}{llll}
\hline & Subway & Bus & Car \\
\hline Subway & $(0,0)$ & (Loss, gain) & (Gain, loss) \\
Bus & (Gain, loss) & $(0,0)$ & (Gain, loss) \\
Car & (Loss, gain) & (Loss, gain) & $(0,0)$ \\
\hline
\end{tabular}

\begin{tabular}{llllllll}
\hline links & $t_{a}^{0}$ (minute) & $C_{a}$ & $\eta_{0}$ & $\eta_{1}$ & $\tau_{a}$ & $E_{a}$ & $F_{a}$ \\
\hline Subway & 30 & - & - & - & $5 \mathrm{RMB} /$ trip & - & - \\
Bus & 45 & 100 & 0.0015 & 2 & $2 \mathrm{RMB} /$ trip & 1 & 3 \\
Car & 10 & 100 & 0.0015 & 2 & $2 \mathrm{RMB} /$ minute & - & - \\
\hline
\end{tabular}


set as $C_{a \in A_{t}^{b}}=50$ and $C_{a \in A_{t}^{s}}=1200$. The parameters in the utility function of work activity are set as: $u_{a}^{\max }=10, \alpha_{a}=720, \beta_{a}=0.02, \gamma_{a}=1$.

\section{Results}

The results of sensitivity analysis of loss aversion on each attribute are shown in Fig. 4. The horizontal axis represents the time of day. For example, 0 stands for 6:00 a.m., and 20 for 7:40 a.m. as one interval is $5 \mathrm{~min}$. The vertical axis denotes the volume of path inflow. Each sub-figures (a)-(c) gives the time-dependent traffic flows on each path of each traveler class when $\beta_{G T}=0.001, \beta_{L T}=-0.005, \beta_{G M}=0.001, \beta_{L M}=-0.005$. For the first class of travelers, the reference being path 1 , most travelers choose paths 1 and 5 at equilibrium state. The reason of choosing path 5 is that it has loss in money but much more gain in time than paths 2, 3 and 4. In particular, path 4 has little gain in money but more loss in time so that choosing path 4 means less path utility. For the second class of travelers, path 4 being the reference, the demand is assigned to 5 paths evenly because, comparing with the reference path, other paths have losses in money but their gains in time are enough to compensate the losses in money, making them close to the reference. And for the third class, only path 5 has traffic flows as other paths' gain in money can't compensate the loss in time; in other words, choosing other paths cannot obtain higher path utilities. Subfigures (d)-(f) shows the path flows when the degree of loss aversion on money increases from 0.005 to 0.05 , which means the constant sensitivity on money is greater than that on time. For the first class, at equilibrium state, most travelers choose path 1 while none

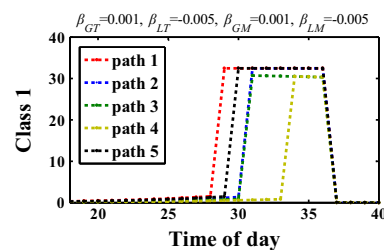

(a)

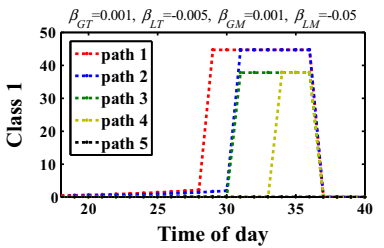

(d)

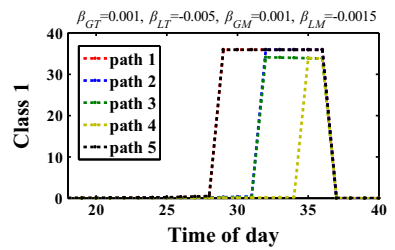

(g)

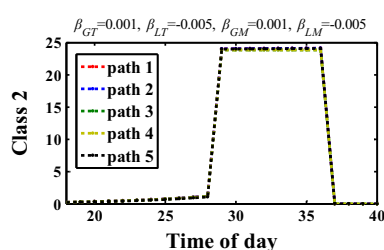

(b)

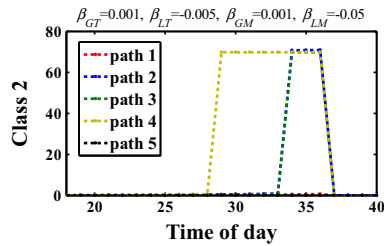

(e)

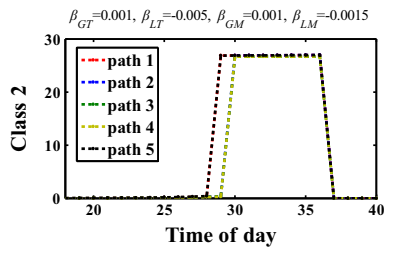

(h)

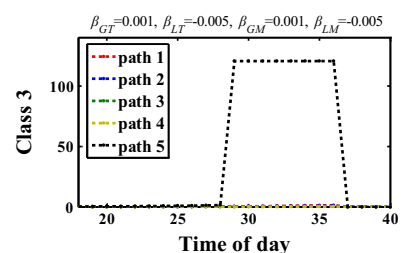

(c)

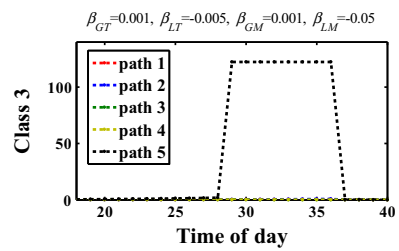

(f)

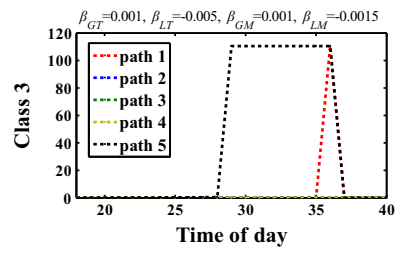

(i)

Fig. 4 Sensitivity analysis of loss aversion in the money and time attribute 
chooses path 5 since it has more loss in money than other paths; for the second class, traffic flows are assigned into paths with the mode of bus, which means no traffic flow on paths 1 and 5 as the increased loss aversion on money makes the utility of paths with subway and car decrease; and for the third class, only path 5 has traffic flows as Fig. 4c. The third row of Fig. 4 shows the path flows when the degree of loss aversion on money loss decreases from 0.005 to 0.0015 compared with the first row of Fig. 4. In this case, travelers' constant sensitivity loom larger on time than money. For the first class, most traffic flows take place on paths 1 and 5 since other paths have more losses in time; for the second class, there is no big differences among the path flows when the reference is path 4, due to the fact that decreasing sensitivity on money makes other paths utility increase compared with Fig. 4e; and for the third class, no traffic flows into paths 2, 3 and 4.

The results above indicate that our model can capture the influence of reference-dependent decision-making mechanism on the activity-travel choices.

In addition, the coefficient of late arrival for activity participation has influence on the departure time choice. Comparing with Fig. 4a-c, in which $\beta_{a}^{L T, l}=-1.5$, Fig. 5 shows the time-dependent traffic flows on each path when increasing $\beta_{a}^{L T, l}$ to -0.0025 . As the penalty decreases, travelers' departure time are postponed.

\section{Comparison analysis}

To examine the effects of reference modes, two additional scenarios are considered. Scenario 1 is based on the principle of utility maximization without any reference, while Scenario 2 represents the case that the reference categories are fixed and exogenous, which have been adopted in static traffic assignment problems (Delle Site and Filippi 2011).

\section{Scenario 1 (no reference)}

In this case, since there is no reference mode, travelers are homogenous and the total travel demand is 3000. Suppose $\beta_{a}^{e}=-\beta_{t}$ and $\beta_{a}^{l}=-1.5$. As Fig. 6a shows, when $\beta_{t}=0.01, \beta_{m}=0.005$, most travelers choose path 5 as their value of time is larger than their value of money, which makes the travel disutilities of taking subway and bus larger than using car. In Fig. 6b, keeping $\beta_{t}$ unchanged and increasing $\beta_{m}$ from 0.005 to 0.05 , it turns out that few travelers use the car to commute. In Fig. $6 \mathrm{c}$, with $\beta_{m}=0.005$ and increasing $\beta_{t}$ from 0.01 to 0.02 , the penalty of early arrival is increased. Travelers taking car depart later than that in Fig. 6a. Compare with Fig. 4, the results show that the utility maximization rule does not capture the loss aversion among the mode alternatives.

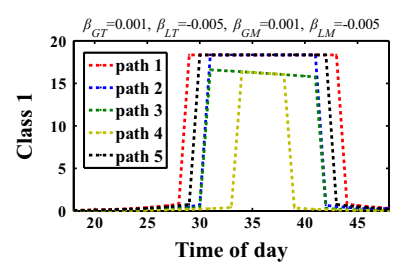

(a)

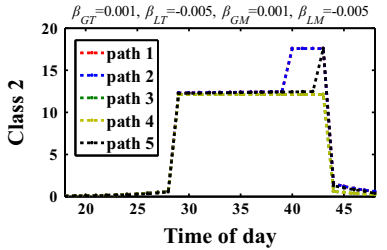

(b)

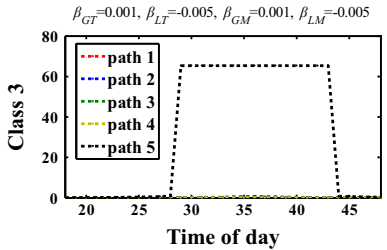

(c)

Fig. 5 Influences of the coefficient of late arrival on the activity-travel path choice 


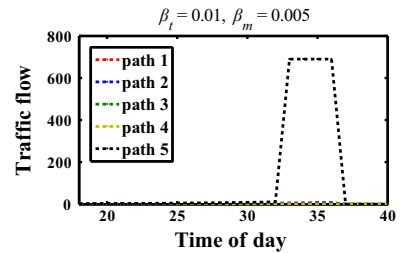

(a)

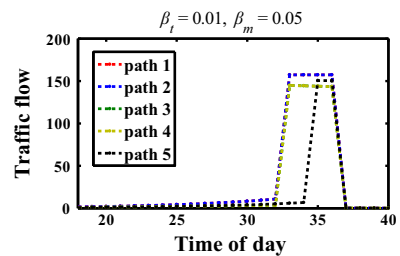

(b)

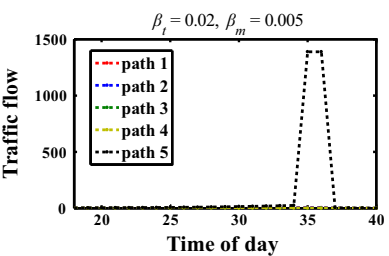

(c)

Fig. 6 Sensitivity analysis under the principle of utility maximization

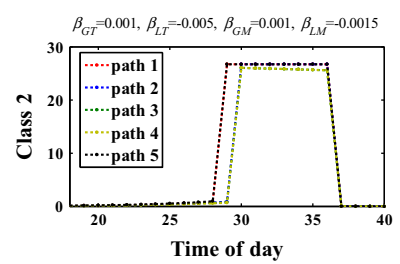

(a) $(45,2)$ as the reference point

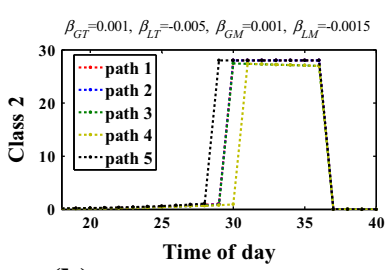

(b) $(50,7)$ as the reference point

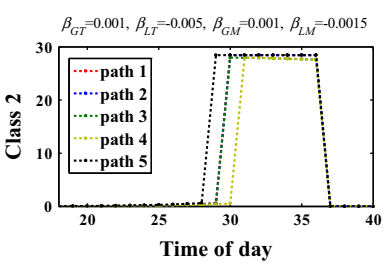

(c) $(60,17)$ as the reference point

Fig. 7 Solution results for fixed and exogenous reference points

\section{Scenario 2 (fixed and exogenous references)}

Figure 7 shows the results when the reference points are fixed and exogenous. The Fig. 7a gives the results of class 2 whose reference point is $(45,2)$ as the free-flow travel time and lowest monetary cost. Traffic flows on paths 1 and 5 are same. Enlarging the value of reference points to $(50,7)$ and $(60,17)$ respectively, as Fig. $7 b$, c show, less traffic flows are on path 1 and little difference between the equilibrium flows under enlarged reference points. It indicates that finding proper reference points in dynamic scheme remains to be a limitation of the model with fixed and exogenous references.

\section{Conclusions}

In this paper, a reference-dependent user equilibrium model is proposed for activity-travel scheduling problem within a simplified multi-state supernetwork representation. Reference- dependent utility theory considers individuals' loss-averse attitude. Travelers are divided into various classes based on their time-dependent and endogenous reference points/categories. A solution algorithm based on route swapping is applied to an illustrative example. The simulated results indicate that reference points and loss aversion attitudes have significant effects on the choice of departure time and mode.

Nevertheless, the current model has some limitations. First, it should be emphasized that we focused on choice-making principles and that the specifications of the utility functions deserves further attention in any applied research. The utility function for activity-travel links is quite flexible; however, the linear functions for travel time and early-late arrival were chosen for convenience. Although linear additive utility functions dominate discrete choice modeling and have been invariably been used in multi-state supernetwork models, non-linear utility functions in theory are better represent actual choice behavior. Second, 
loss aversion has been assumed invariant in the current model specification, but in reality it is context-dependent. Reference points with regard to departure times depend on the nature of activities and on travel party, while in addition travelers differ in their decision-making behavior. Third, although the current model has been inspired by the consideration of near to perfect information, justifying the deterministic representation used in the current model, equivalent stochastic formulations should be develop. It is due to the credibility of the information and the fact that multi-state supernetworks are concerned with full daily schedules and consequently varying degree of uncertainty in the information provision may exude. Finally, the activity-travel patterns in the current study are simplified extractions from the multi-state supernetwork representations. Extending the suggested referencedependent user equilibrium to multi-modal and multi-activity trip chains will make our model more practical. Most of these issues can be viewed as extensions and elaborations of the suggested model and can be solved with the solution algorithm. Addressing these limitations requires additional original work. Work along these lines will be reported in future publications.

Acknowledgments This work was jointly supported by the Netherlands Organization for Scientific Research (NWO) and the National Natural Science Foundation of China (Project No. 71371094, 71471084).

Open Access This article is distributed under the terms of the Creative Commons Attribution 4.0 International License (http://creativecommons.org/licenses/by/4.0/), which permits unrestricted use, distribution, and reproduction in any medium, provided you give appropriate credit to the original author(s) and the source, provide a link to the Creative Commons license, and indicate if changes were made.

\section{References}

Arentze, T.A., Timmermans, H.J.P.: Multistate supernetwork approach to modelling multi-activity, multimodal trip chains. Int. J. Geogr. Inf. Sci. 18(7), 631-651 (2004)

Bovy, P.H., Hoogendoorn-Lanser, S.: Modelling route choice behaviour in multi-modal transport networks. Transportation 32(4), 341-368 (2005)

De Borger, B., Fosgerau, M.: The trade-off between money and travel time: a test of the theory of referencedependent preferences. J. Urban Econ. 64(1), 101-115 (2008)

Delle Site, P., Filippi, F.: Stochastic user equilibrium and value-of-time analysis with reference-dependent route choice. EJTIR 2(11), 194-218 (2011)

Delle Site, P., Filippi, F., Castaldi, C.: Reference-dependent stochastic user equilibrium with endogenous reference points. EJTIR 13(2), 147-168 (2013)

Ettema, D., Timmermans, H.J.P.: Modeling departure time choice in the context of activity scheduling behavior. Transp. Res. Rec. 1831, 39-46 (2003)

Fu, X., Lam, W.H.: A network equilibrium approach for modelling activity-travel pattern scheduling problems in multi-modal transit networks with uncertainty. Transportation 41(1), 37-55 (2014)

Huang, H.J., Lam, W.H.: Modeling and solving the dynamic user equilibrium route and departure time choice problem in network with queues. Transp. Res. B 36(3), 253-273 (2002)

Kahneman, D., Tversky, A.: Prospect theory: an analysis of decision under risk. Econometrica 47(2), 263-291 (1979)

Lam, W.H., Li, Z.C., Huang, H.J., Wong, S.C.: Modeling time-dependent travel choice problems in road networks with multiple user classes and multiple parking facilities. Transp. Res. B 40(5), 368-395 (2006)

Li, Z.C., Lam, W.H., Wong, S.C., Sumalee, A.: An activity-based approach for scheduling multimodal transit services. Transportation 37(5), 751-774 (2010)

Liao, F.: Modeling duration choice in space-time multi-state supernetworks for individual activity-travel scheduling. Transp. Res. C 69, 16-35 (2016)

Liao, F., Arentze, T., Timmermans, H.J.P.: Supernetwork approach for multimodal and multi-activity travel planning. Transp. Res. Rec. 2175, 38-46 (2010) 
Liao, F., Arentze, T.A., Timmermans, H.J.P.: Constructing personalized transportation networks in multistate supernetworks: a heuristic approach. Int. J. Geogr. Inf. Sci. 25(11), 1885-1903 (2011)

Liao, F., Arentze, T., Timmermans, H.J.P.: Incorporating space-time constraints and activity-travel time profiles in a multi-state supernetwork approach to individual activity-travel scheduling. Transp. Res. B 55, 41-58 (2013a)

Liao, F., Arentze, T.A., Timmermans, H.J.P.: Multi-state supernetwork framework for the two-person joint travel problem. Transportation 40(4), 813-826 (2013b)

Liu, P., Liao, F., Huang, H.J., Timmermans, H.J.P.: Dynamic activity-travel assignment in multi-state supernetworks. Transp. Res. B 81, 656-671 (2015)

Lo, H.K., Yip, C.W., Wan, K.H.: Modeling transfer and non-linear fare structure in multi-modal network. Transp. Res. B 37(2), 149-170 (2003)

Nagurney, A., Zhang, D.: Projected dynamical systems in the formulation, stability analysis, and computation of fixed-demand traffic network equilibria. Transp. Sci. 31(2), 147-158 (1997)

Ouyang, L.Q., Lam, W.H., Li, Z.C., Huang, D.: Network user equilibrium model for scheduling daily activity travel patterns in congested networks. Transp. Res. Rec. 2254(1), 131-139 (2011)

Pinjari, A.R., Bhat, C.R.: Activity-based travel demand analysis. In: de Palma, A. (ed.) A Handbook of Transport Economics, chap. 10, pp. 213-248. Edward Elgar Publishing, Cheltenham (2011)

Ramadurai, G., Ukkusuri, S.: Dynamic user equilibrium model for combined activity-travel choices using activity-travel supernetwork representation. Netw. Spat. Econ. 10(2), 273-292 (2010)

Rasouli, S., Timmermans, H.J.P.: Activity-based models of travel demand: promises, progress and prospects. Int. J. Urban Sci. 18(1), 31-60 (2014)

Senbil, M., Kitamura, R.: Reference points in commuter departure time choice: a prospect theoretic test of alternative decision frames. Intell. Transp. Syst. 8(1), 19-31 (2004)

Szeto, W.Y., Lo, H.K.: Dynamic traffic assignment: properties and extensions. Transportmetrica 2(1), 31-52 (2006)

Tversky, A., Kahneman, D.: Loss aversion in riskless choice: a reference-dependent model. Quart. J. Econ. 106, 1039-1061 (1991)

Qing $\mathbf{L i}$ is a Ph.D. candidate of School of Management and Engineering at Nanjing University (China) and currently a visiting scholar at the Urban Planning Group, Eindhoven University of Technology (the Netherlands). Her work mainly focuses on modeling activity-based dynamic traffic assignment problems with the consideration of various decision-making mechanisms.

Feixiong Liao is currently an assistant professor at the Urban Planning Group, Eindhoven University of Technology. His research interests include activity-based modelling, accessibiity analysis, discrete choice modelling, and supernetwork modelling for applications in transportation research and urban planning.

Harry J. P. Timmermans is chair professor of Urban Planning at the Eindhoven University of Technology. His research interest concerns the analysis and modeling of consumer preferences and choice behavior in a variety of application contexts and the development and application of decision support systems.

Jing Zhou is a professor of School of Management and Engineering at Nanjing University. Her research interests mainly focus on the modeling of traffic assignment problems. 